\title{
Low Probability of Intercept Triangular Modulated Frequency Modulated Continuous Wave Signal Characterization Comparison using the Wigner Ville Distribution and the Choi Williams Distribution
}

By Daniel L. Stevens Abstract-Digital intercept receivers are currently moving away from Fourier-based analysis and towards classical timefrequency analysis techniques for the purpose of analyzing low probability of intercept radar signals. This paper presents the novel approach of characterizing low probability of intercept triangular modulated frequency modulated continuous wave radar signals through utilization and direct comparison of the Wigner Ville Distribution versus the Choi Williams Distribution. The following metrics were used for evaluation: percent error of: carrier frequency, modulation bandwidth, modulation period, chirp rate, and time-frequency localization ( $x$ and $y$ direction). Also used were: percent detection, lowest signal-tonoise ratio for signal detection, and plot (processing) time. Experimental results demonstrate that overall, the Wigner Ville Distribution produced more accurate characterization metrics than the Choi Williams Distribution. An improvement in performance may well translate into an increase in personnel safety.

Strictly as per the compliance and regulations of:

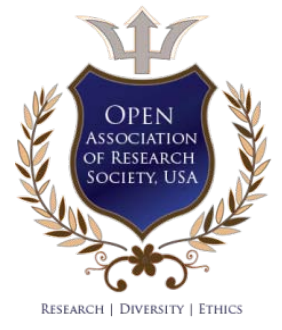

(C) 2019. Daniel L. Stevens. This is a research/review paper, distributed under the terms of the Creative Commons AttributionNoncommercial 3.0 Unported License http://creativecommons.org/licenses/by-nc/3.0/), permitting all non commercial use, distribution, and reproduction in any medium, provided the original work is properly cited. 


\section{Low Probability of Intercept Triangular Modulated Frequency Modulated Continuous Wave Signal Characterization Comparison using the Wigner Ville Distribution and the Choi Williams Distribution}

Daniel L. Stevens

\begin{abstract}
Digital intercept receivers are currently moving away from Fourier-based analysis and towards classical timefrequency analysis techniques for the purpose of analyzing low probability of intercept radar signals. This paper presents the novel approach of characterizing low probability of intercept triangular modulated frequency modulated continuous wave radar signals through utilization and direct comparison of the Wigner Ville Distribution versus the Choi Williams Distribution. The following metrics were used for evaluation: percent error of: carrier frequency, modulation bandwidth, modulation period, chirp rate, and time-frequency localization $(x$ and $y$ direction). Also used were: percent detection, lowest signal-tonoise ratio for signal detection, and plot (processing) time. Experimental results demonstrate that overall, the Wigner Ville Distribution produced more accurate characterization metrics than the Choi Williams Distribution. An improvement in performance may well translate into an increase in personnel safety.
\end{abstract}

\section{INTRODUCTION}

requency Modulated Continuous Wave (FMCW) signals are frequently encountered in modern radar systems [WAN10], [WON09], [WAJ08]. The frequency modulation spreads the transmitted energy over a large modulation bandwidth $\Delta F$, providing good range resolution that is critical for discriminating targets from clutter. The power spectrum of the FMCW signal is nearly rectangular over the modulation bandwidth, so non-cooperative interception is difficult. Since the transmit waveform is deterministic, the form of the return signals can be predicted. This gives it the added advantage of being resistant to interference (such as jamming), since any signal not matching this form can be suppressed [WIL06]. Consequently, it is difficult for an intercept receiver to detect the FMCW waveform and measure the parameters accurately enough to match the jammer waveform to the radar waveform [PAC09].

The most popular linear modulation utilized is the triangular FMCW emitter [LIA09], since it can

Author: Air Force Research Laboratory Rome.

e-mail: daniel.stevens.7@us.af.mil measure the target's range and Doppler [MIL02], [LIW08]. Triangular modulated FMCW is the waveform that is employed in this paper.

Time-frequency signal analysis involves the analysis and processing of signals with time-varying frequency content. Such signals are best represented by a time-frequency distribution [PAP95], [HAN00], which is intended to show how the energy of the signal is distributed over the two-dimensional time-frequency plane [WEI03], [LIX08], [OZD03]. Processing of the signal may then exploit the features produced by the concentration of signal energy in two dimensions (time and frequency), instead of only one dimension (time or frequency) [BOA03], [LIY03]. Since noise tends to spread out evenly over the time-frequency domain, while signals concentrate their energies within limited time intervals and frequency bands; the local SNR of a 'noisy' signal can be improved simply by using time-frequency analysis [XIA99]. Also, the intercept receiver can increase its processing gain by implementing timefrequency signal analysis [GUL08].

Time-frequency distributions are useful for the visual interpretation of signal dynamics [RAN01]. An experienced operator can quickly detect a signal and extract the signal parameters by analyzing the timefrequency distribution [ANJ09].

Wigner Ville Distribution (WVD)

One of the most prominent members of the time-frequency analysis techniques family is the WVD. The WVD satisfies a large number of desirable mathematical properties. In particular, it is always realvalued, preserves time and frequency shifts, and satisfies marginal properties [QIA02]. The WVD, which is a transformation of a continuous time signal into the time-frequency domain, is computed by correlating the signal with a time and frequency translated version of itself, making it bilinear. The WVD exhibits the highest signal energy concentration in the time-frequency plane [WIL06]. By using the WVD, an intercept receiver can come close to having a processing gain near the LPI radar's matched filter processing gain [PAC09]. The 
WVD also contains cross term interference between every pair of signal components, which may limit its applications [GUL07], [STE96], and which can make the WVD time-frequency representation hard to read, especially if the components are numerous or close to each other, and the more so in the presence of noise [BOA03]. This lack of readability can in turn translate into decreased signal detection and parameter extraction metrics, potentially placing the intercept receiver signal analyst's platform in harm's way.

The WVD of a signal $x(t)$ is given in equation (1) as:

$$
W_{x}(t, f)=\int_{-\infty}^{+\infty} x\left(t+\frac{\tau}{2}\right) x^{*}\left(t-\frac{\tau}{2}\right) e^{-j 2 \pi f \tau} d \tau
$$

or equivalently in equation (2) as:

$$
W_{x}(t, f)=\int_{-\infty}^{+\infty} X\left(f+\frac{\xi}{2}\right) X^{*}\left(f-\frac{\xi}{2}\right) e^{j 2 \pi \xi t} d \xi
$$

\section{Choi Williams Distribution (CWD)}

The CWD is a member of the Cohen's class of time-frequency distributions which use smoothing kernels [GUL07] to help reduce cross-term interference so prevalent in the WVD [BOA03], [PAC09], [UPP08]. The reduction in cross-term interference can make the time-frequency representation more readable and can make signal detection and parameter extraction more accurate. The down-side is that the CWD, like all members of Cohen's class, is faced with an inevitable trade-off between cross-term reduction and timefrequency localization. Because of this, the signal detection and parameter extraction benefits gained by the cross-term reduction may be offset by the decrease in time-frequency localization (smearing or widening of the signal).

The CWD of a signal $x(s)$ is given in equation (3) as:

$$
C W_{x}(t, f)=\sqrt{\frac{2}{\pi}} \iint_{-\infty}^{+\infty} \frac{\sigma}{|\tau|} e^{-2 \sigma^{2}(s-t)^{2} / \tau^{2}} x\left(s+\frac{\tau}{2}\right) x^{*}\left(s-\frac{\tau}{2}\right) e^{-j 2 \pi f \tau} d s d \tau
$$

As can be seen from equation (3), the CWD uses an exponential kernel in the generalized class of bilinear time-frequency distributions. Choi and Williams introduced one of the earliest 'new' distributions [CHO89], which they called the Exponential Distribution or ED. This new distribution overcomes several drawbacks of the Spectrogram and the WVD, providing a good trade-off between localization and suppressed interferences [WIL92], [GUL07], [UPP08]. Interference terms tend to lie away from the axes in the ambiguity plane, while auto terms (signals) tend to lie on the axes. The Spectrogram kernel attenuates everything away from the $(0,0)$ point, the WVD kernel passes everything, and the CWD kernel passes everything on the axes and attenuates away from the axes. Thus, the CWD generally attenuates interference terms [PAC09], [HLA92]. This provides its reduced interference characteristic. The Spectrogram reduces interference also, but at a cost to the signal concentration.

\section{Methodology}

The methodologies detailed in this section describe the processes involved in obtaining and comparing metrics between the classical time-frequency analysis techniques of the Wigner Ville Distribution and the Choi Williams Distribution for the detection and characterization of low probability of intercept triangular modulated FMC Wradar signals.
The tools used for this testing were: MATLAB (version 8.3), Signal Processing Toolbox (version 6.21), Wavelet Toolbox (version 4.13), Image Processing Toolbox (version 9.0), Time-Frequency Toolbox (version 1.0) (http://tftb.nongnu.org/).

All testing was accomplished on a desktop computer (Dell Precision T1700; Processor -Intel Xeon CPU E3-1226 v3 3.30GHz; Installed RAM - 32.0GB; System type - 64-bit operating system, x64-based processor)

Testing was performed for the triangular modulated FMCW waveform, whose parameters were chosen for academic validation of signal processing techniques. Due to computer processing resources they were not meant to represent real-world values. The number of samples was chosen to be either 256 or 512 , which seemed to be the optimum size for the desktop computer. Testing was performed at three different SNR levels: $10 \mathrm{~dB}, 0 \mathrm{~dB}$, and the lowest SNR at which the signal could be detected. The noise added was white Gaussian noise, which best reflects the thermal noise present in the IF section of an intercept receiver [PAC09]. Kaiser windowing was used, when windowing was applicable. 100 runs were performed for each test, for statistical purposes. The plots included in this paper were done at a threshold of $5 \%$ of the maximum intensity and were linear scale (not $\mathrm{dB}$ ) of analytic (complex) signals; the color bar represented intensity. 
The signal processing tools used for each task were the Wigner Ville Distribution and the Choi Williams Distribution.

The triangular modulated FMCW signal (most prevalent LPI radar waveform [LIA09]) used had the following parameters: sampling frequency $=4 \mathrm{KHz}$; carrier frequency $=1 \mathrm{KHz}$; modulation bandwidth = $500 \mathrm{~Hz}$; modulation period $=.02 \mathrm{sec}$.

After each particular run of each test, metrics were extracted from the time-frequency representation. The different metrics extracted were as follows:

\section{b) Percent detection}

Percent of time signal was detected - signal was declared a detection if any portion of each of the signal components (4 chirp components for triangular modulated FMCW) exceeded a set threshold (a certain percentage of the maximum intensity of the timefrequency representation).

Threshold percentages were determined based on visual detections of low SNR signals (lowest SNR at which the signal could be visually detected in the timefrequency representation) (see Figure 1).

\section{a) Plot (processing) time}

Time required for plot to be displayed.

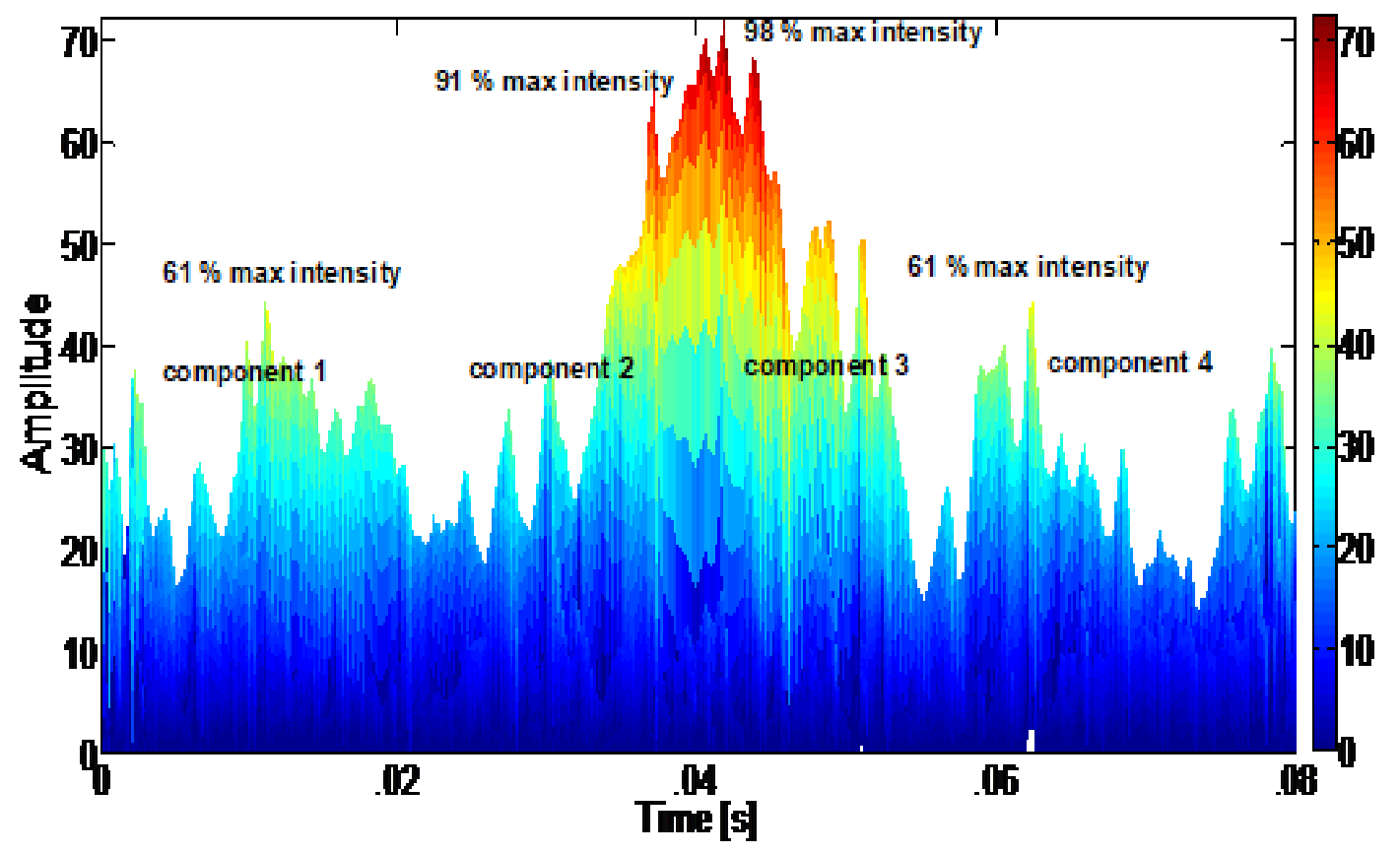

Figure 1: Threshold percentage determination. This plot is a time vs. amplitude ( $\mathrm{x}-\mathrm{z}$ view) of the CWD of a triangular modulated FMCW signal (256 samples, with $S N R=-3 d B$ ). For visually detected low SNR plots (like this one), the percent of max intensity for the peak z-value of each of the signal components (the 2 legs for each of the 2 triangles of the triangular modulated FMCW) was noted (here 61\%,91\%,98\%,61\%), and the lowest of these 4 values was recorded (61\%). Ten test runs were performed for both time-frequency analysis tools (CWD and WVD) for this waveform. The average of these recorded low values was determined and then assigned as the threshold for that particular time-frequency analysis tool. Note - the threshold for the CWD is $60 \%$.

Thresholds were assigned as follows: CWD (60\%); WVD (50\%).

For percent detection determination, these threshold values were included in the time-frequency plot algorithms so that the thresholds could be applied automatically during the plotting process. From the threshold plot, the signal was declared a detection if any portion of each of the signal components was visible (see Figure 2). 


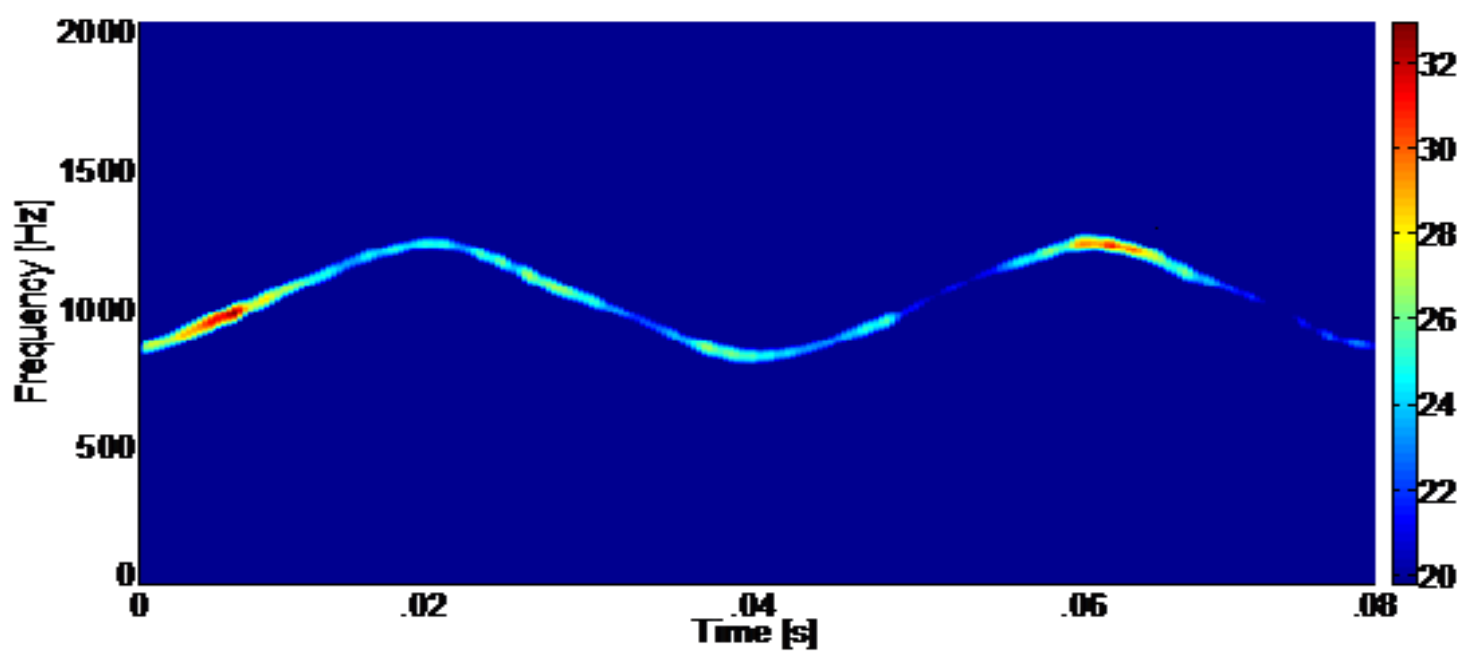

Figure 2: Percent detection (time-frequency). This plot is a time vs. frequency ( $x-y$ view) of the CWD of a triangular modulated FMCW signal (256 samples, with $\mathrm{SNR}=10 \mathrm{~dB}$ ) with threshold value automatically set to $60 \%$. From this threshold plot, the signal was declared a (visual) detection because at least a portion of each of the 4 signal components (the 2 legs for each of the 2 triangles of the triangular modulated FMCW) was visible.

c) Carrier frequency Figure 3).

The frequency corresponding to the maximum intensity of the time - frequency representation (see

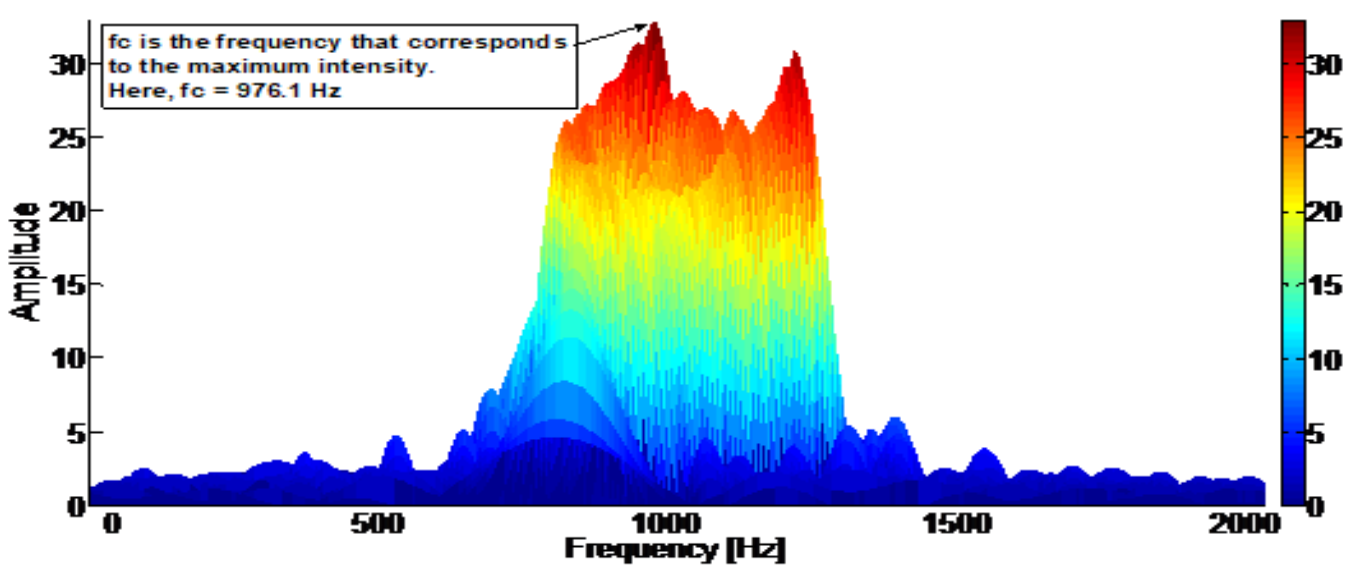

Figure 3: Determination of carrier frequency. CWD of a triangular modulated FMCW signal (256 samples, $S N R=10 \mathrm{~dB}$ ). From the frequency vs. amplitude ( $\mathrm{y}-\mathrm{z}$ view), the maximum intensity value is manually determined. The frequency corresponding to the max intensity value is the carrier frequency (here $\mathrm{fc}=976.1 \mathrm{~Hz}$ ).

\section{d) Modulation bandwidth}

Distance from highest frequency value of signal (at a threshold of $20 \%$ maximum intensity) to lowest frequency value of signal (at same threshold) in $\mathrm{Y}$ direction (frequency).

The threshold percentage was determined based on manual measurement of the modulation bandwidth of the signal in the time-frequency representation. This was accomplished for ten test runs of each time-frequency analysis tool (Wigner Ville Distribution and Choi Williams Distribution), for the triangular modulated FMCW waveform. During each manual measurement, the max intensity of the high and low measuring points was recorded. The average of the max intensity values for these test runs was $20 \%$. This was adopted as the threshold value, and is representative of what is obtained when performing manual measurements. This $20 \%$ threshold was also adapted for determining the modulation period and the time-frequency localization (both are described below).

For modulation bandwidth determination, the $20 \%$ threshold value was included in the time-frequency plot algorithms so that the threshold could be applied automatically during the plotting process. From the 
threshold plot, the modulation bandwidth was manually

measured (see Figure 4).

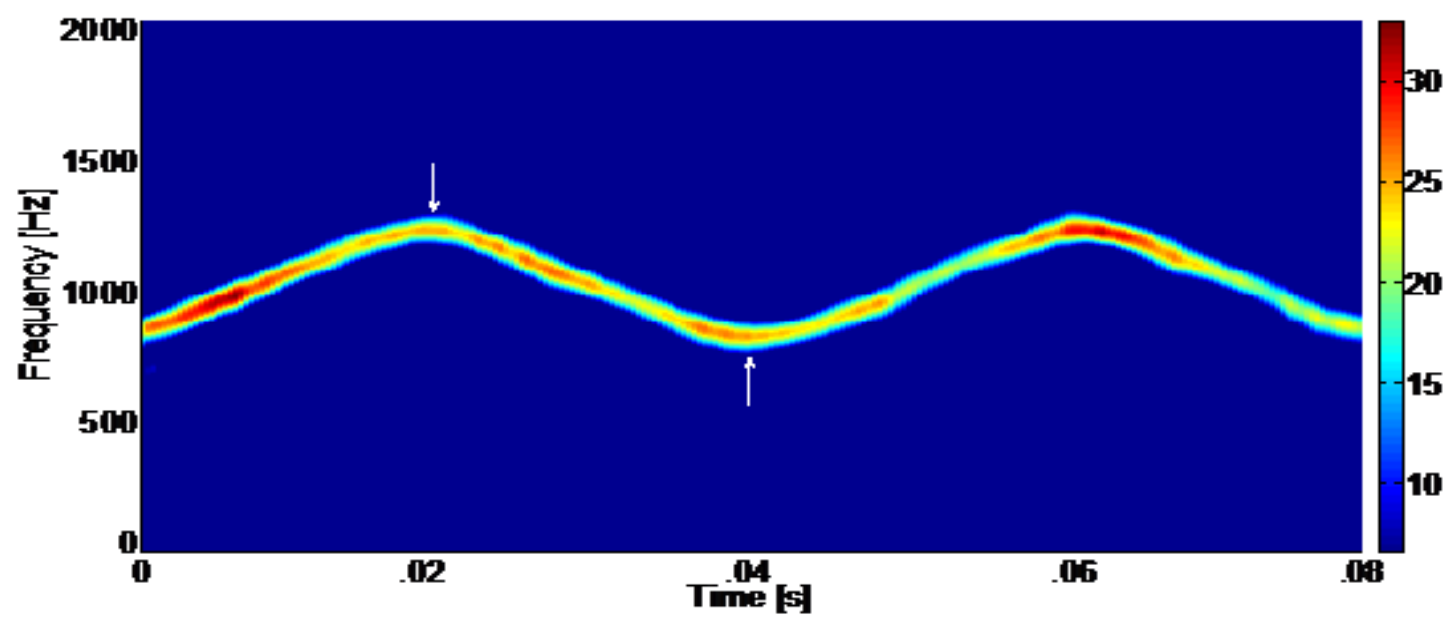

Figure 4: Modulation bandwidth determination. This plot is a time vs. frequency ( $x-y$ view) of the CWD of a triangular modulated FMCW signal (256 samples, SNR=10dB) with threshold value automatically set to $20 \%$. From this threshold plot, the modulation bandwidth was measured manually from the highest frequency value of the signal (top white arrow) to the lowest frequency value of the signal (bottom white arrow) in the y_direction (frequency).

e) Modulation period

Distance from highest frequency value of signal (at a threshold of $20 \%$ maximum intensity) to lowest frequency value of signal (at same threshold) in Xdirection (time).
For modulation period determination, the $20 \%$ threshold value was included in the time-frequency plot algorithms so that the threshold could be applied automatically during the plotting process. From the threshold plot, the modulation period was manually measured (see Figure 5).

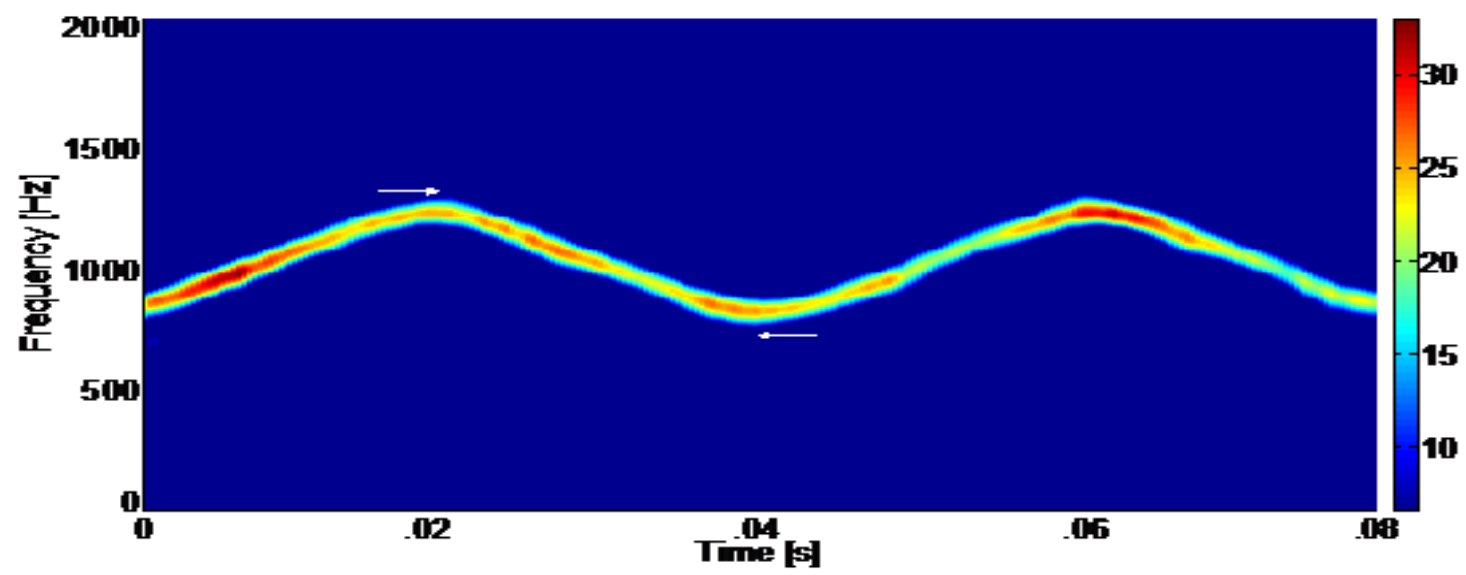

Figure 5: Modulation period determination. This plot is a time vs. frequency (x-y view) of the CWD of a triangular modulated FMCW signal (256 samples, SNR=10dB) with threshold value automatically set to $20 \%$. From this threshold plot, the modulation period was measured manually from the highest frequency value of the signal (top white arrow) to the lowest frequency value of the signal (bottom white arrow) in the $\mathrm{x}$-direction (time).

f) Time-frequency localization

Measure of the thickness of a signal component (at a threshold of $20 \%$ maximum intensity on each side of the component) - converted to \% of entire $X$-Axis, and \% of entire Y-Axis.

For time-frequency localization determination, the $20 \%$ threshold value was included in the time- frequency plot algorithms so that the threshold could be applied automatically during the plotting process. From the threshold plot, the time-frequency localization was manually measured (see Figure 6). 


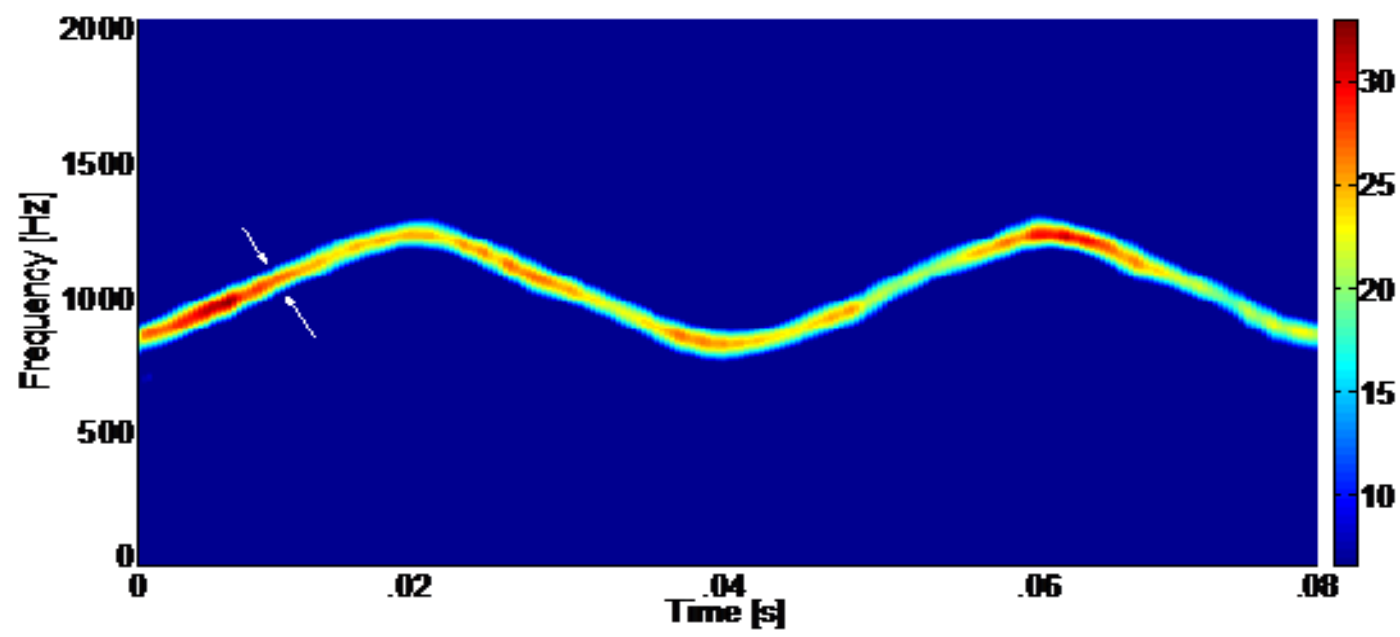

Figure 6: Time-frequency localization determination. This plot is a time vs. frequency ( $x-y$ view) of the CWD of a triangular modulated FMCW signal (256 samples, $\mathrm{SNR}=10 \mathrm{~dB}$ ) with threshold value automatically set to $20 \%$. From this threshold plot, the time-frequency localization was measured manually from the left side of the signal (left white arrow) to the right side of the signal (right white arrow) in both the $\mathrm{x}$-direction (time) and the $y$-direction (frequency). Measurements were made at the center of each of the 4 'legs', and the average values were determined. Average time and frequency 'thickness' values were then converted to: \% of entire $x$-axis and \% of entire $y$-axis.

g) Chirp rate

(modulation bandwidth)/(modulation period)

h) Lowest detectable SNR

The lowest SNR level at which at least a portion of each of the signal components exceeded the set threshold listed in the percent detection section above.

For lowest detectable SNR determination, these threshold values were included in the time-frequency plot algorithms so that the thresholds could be applied automatically during the plotting process. From the threshold plot, the signal was declared a detection if any portion of each of the signal components was visible. The lowest SNR level for which the signal was declared a detection is the lowest detectable SNR (see Figure 7).

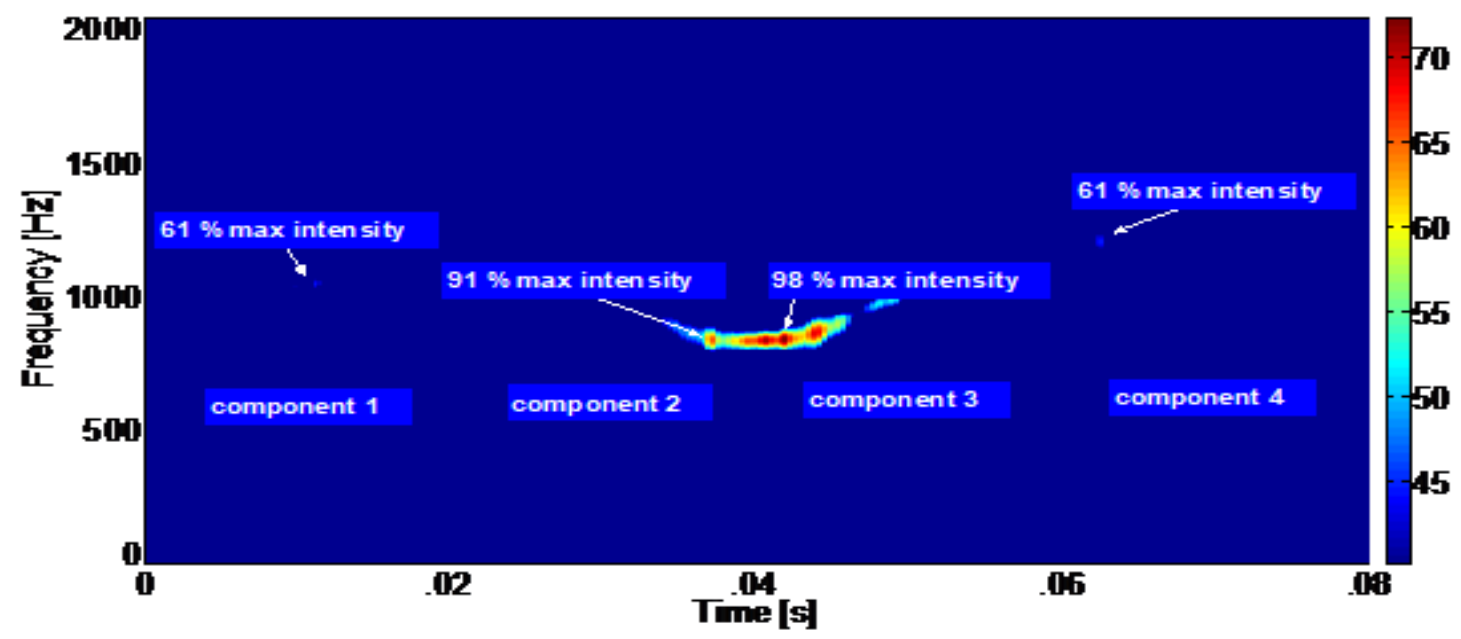

Figure 7: Lowest detectable SNR. This plot is a time vs. frequency ( $\mathrm{x}-\mathrm{y}$ view) of the CWD of a triangular modulated FMCW signal (256 samples, with $S N R=-3 d B$ ) with threshold value automatically set to $60 \%$. From this threshold plot, the signal was declared a (visual) detection because at least a portion of each of the 4 signal components (the 2 legs for each of the 2 triangles of the triangular modulated FMCW) was visible. Note that the signal portion for the two 61\% max intensities are barely visible, because the threshold for the CWD is 60\%. For this case, any lower SNR than $-3 \mathrm{~dB}$ would have been a non-detect. 
The data from all 100 runs for each test was used to produce the actual, error, and percent error for each of these metrics listed above.

The metrics from the Wigner Ville Distribution were then compared to the metrics from the Choi Williams Distribution. By and large, the Wigner Ville Distribution outperformed the Choi Williams Distribution, as will be shown in the results section.

\section{Results}

Table 1 presents the overall test metrics for the two classical time-frequency analysis techniques used in this testing (Choi Williams Distribution versus Wigner Ville Distribution).

Table 1: Overall test metrics (average percent error: carrier frequency, modulation bandwidth, modulation period, chirp rate; average: percent detection, lowest detectable snr, plot time, time-frequency localization (as a percent of $x$ axis and y axis) for the two classical time-frequency analysis techniques (Choi Williams Distribution versus Wigner Ville Distribution).

\begin{tabular}{|c|c|c|}
\hline Parameters & Choi Williams Distribution & Wigner Ville Distribution \\
\hline carrier frequency & $5.22 \%$ & $2.23 \%$ \\
\hline modulation bandwidth & $9.61 \%$ & $5.53 \%$ \\
\hline modulation period & $0.49 \%$ & $0.48 \%$ \\
\hline chirp rate & $9.67 \%$ & $5.28 \%$ \\
\hline percent detection & $69.6 \%$ & $77.6 \%$ \\
\hline lowest detectable snr & $-3.0 \mathrm{db}$ & $-2.3 \mathrm{db}$ \\
\hline plot time & $10.44 \mathrm{~s}$ & $1341.84 \mathrm{~s}$ \\
\hline time-frequency localization-x & $1.89 \%$ & $0.62 \%$ \\
\hline time-frequency localization-y & $3.52 \%$ & $1.28 \%$ \\
\hline
\end{tabular}

From Table 1, the WVD outperformed the CWD in average percent error: carrier frequency $(2.23 \%$ vs. $5.22 \%)$, modulation bandwidth (5.53\% vs. $9.61 \%)$, modulation period $(0.48 \%$ vs. $0.49 \%)$, and chirp rate (5.28\% vs. 9.67\%). The WVD also outperformed the CWD in average: percent detection (77.6\% vs. 69.6\%), time-frequency localization (x-direction) $(0.62 \%$ vs. $1.89 \%)$, and time-frequency localization (y-direction) (1.28\% vs. $3.52 \%)$. The CWD outperformed the WVD in average: lowest detectable SNR (-3.0db vs. $-2.3 \mathrm{db})$ and average plot time (10.44s vs. 1341.84s).

Figure 8 shows comparative plots of the Choi Williams Distribution (left) vs. the Wigner Ville Distribution (right) (triangular modulated FMCW signal) at SNRs of 10dB (top row), OdB (middle row), and $-3 \mathrm{~dB}$ (bottom row).
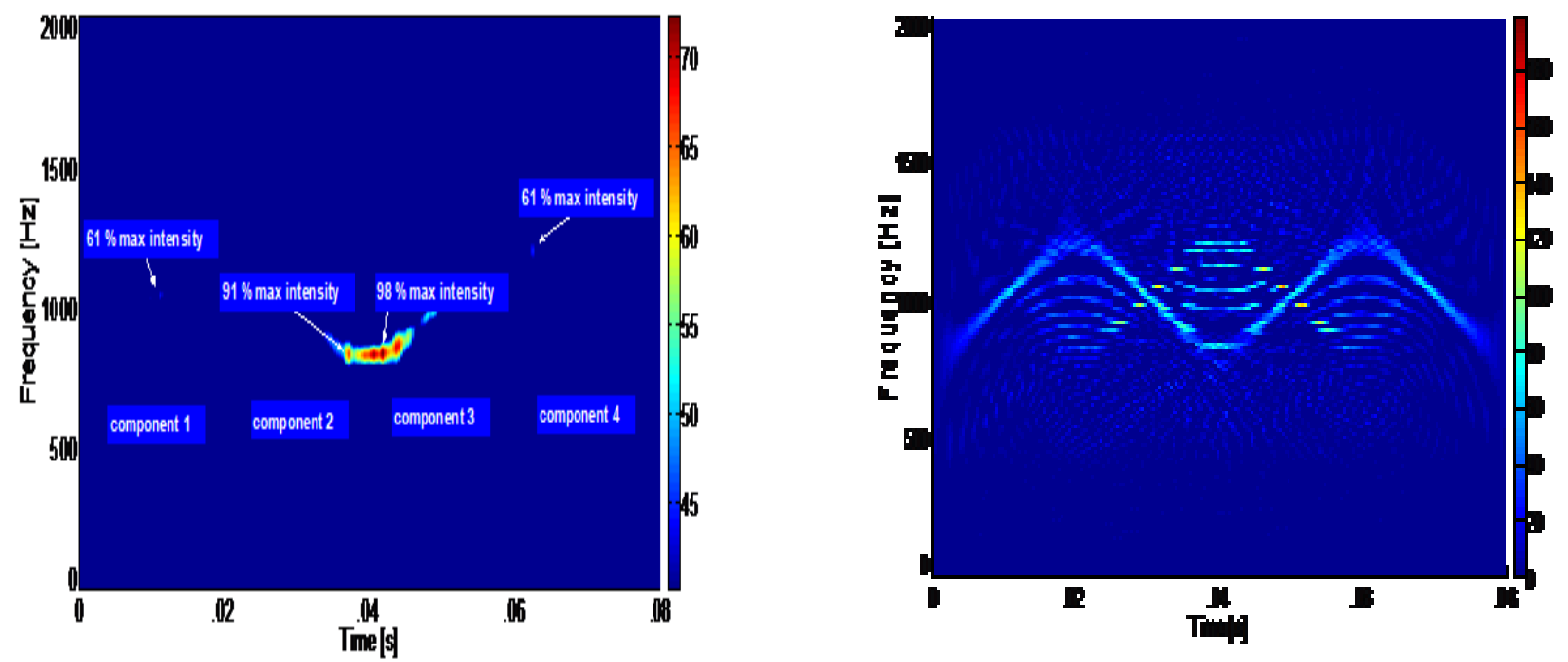

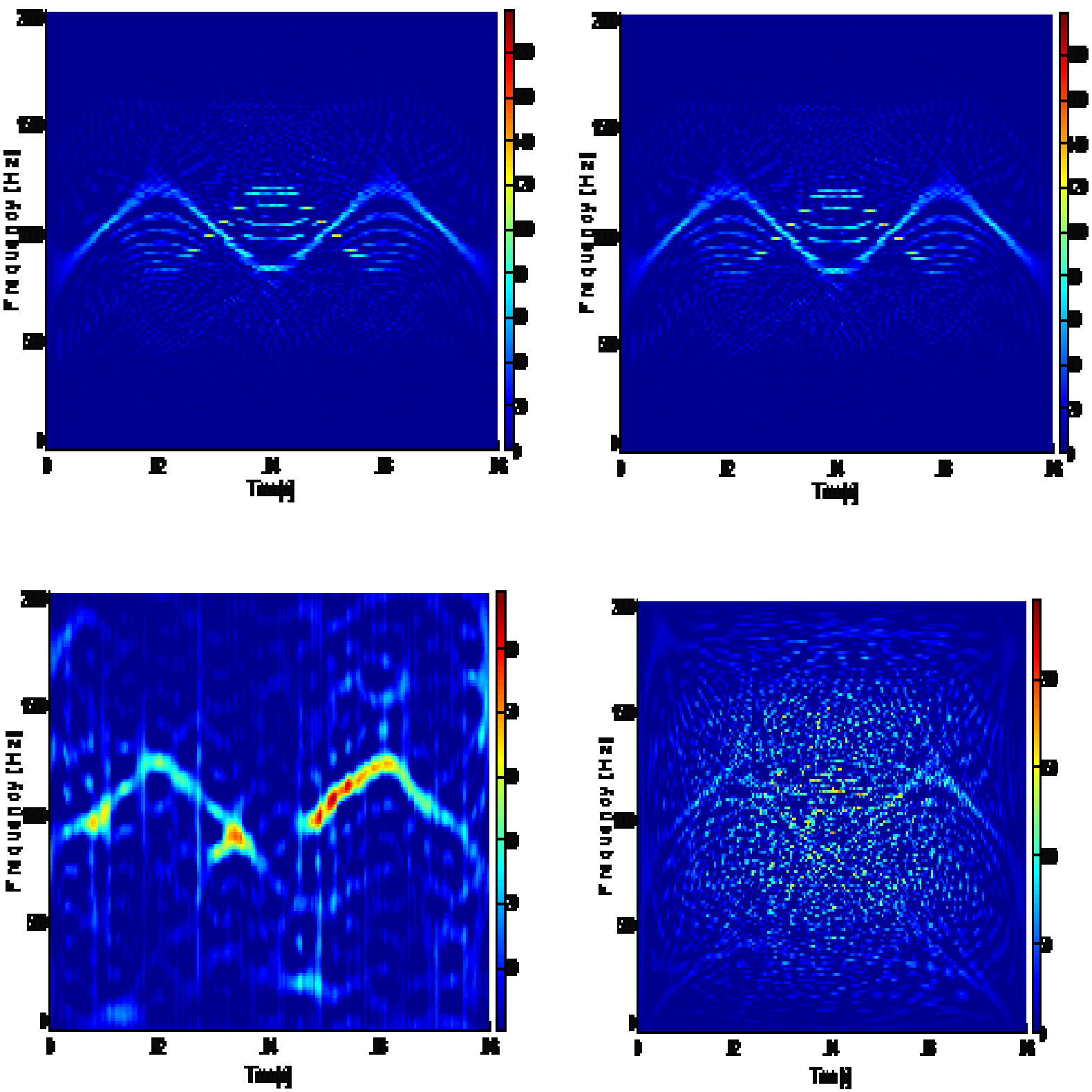

Figure 8: Comparative plots of the triangular modulated FMCWlow probability of intercept radar signals (CWD (lefthand side) vs. the WVD (right-hand side)). The SNR for the top row is $10 \mathrm{~dB}$, for the middle row is 0dB, and for the bottom row is -3dB.In general, the WVD signalappears more localized ('thinner') than does the CWD signal, however, the cross-term interference in the WVDplots makes it more difficult to differentiate betweeen the signal and the cross-term interference.

\section{DISCUSSION}

This section will elaborate on the results from the previous section.

From Table 1, the WVD outperformed the CWD in average percent error: carrier frequency $(2.23 \%$ vs. $5.22 \%)$, modulation bandwidth (5.53\% vs. 9.61\%), modulation period $(0.48 \%$ vs. $0.49 \%)$, and chirp rate (5.28\% vs. $9.67 \%)$; and in average: percent detection
(77.6\% vs. $69.6 \%)$, time-frequency localization ( $\mathrm{x}$ direction) $(0.62 \%$ vs. $1.89 \%)$, and time-frequency localization (y-direction) (1.28\% vs. 3.52\%). These results are by and large a result of the WVD signal being much more localized signal than the CWD signal. The CWD's 'thicker' signal is a result of its cross-term reduction - at the expense of signal localization. 
The CWD outperformed the WVD in average: lowest detectable SNR (-3.0db vs. -2.3db) and average plot time (10.44s vs. 1341.84s). The combination of the CWD's reduction of cross-term interference along with the WVD being very computationally complex [MIL02] are the grounds for the CWD's better plot time. In addition, lowest detectable SNR is based on visual detection in the Time-Frequency representation. Figure 8 shows that, for the WVD plots, as the SNR gets lower, it becomes more difficult to distinguish between the actual signal and the cross-term interference. However, for the CWD plots there is no cross-term interference to confuse with the actual signals, making the CWD signal, though not as localized, more easily detected than the WVD signal - at these lower SNRs.

The WVD might be used in a scenario where you need good signal localization in a fairly low SNR environment, without tight time constraints. The CWD might be used in a scenario where a short plot time is necessary, and where signal localization is not an issue. Such a scenario might be a 'quick and dirty' check to see if a signal is present, without precise extraction of its parameters.

\section{Conclusions}

Digital intercept receivers, whose main job is to detect and extract parameters from low probability of intercept radar signals, are currently moving away from Fourier-based analysis and towards classical timefrequency analysis techniques, such as the Wigner Ville Distribution, and the Choi Williams Distribution, for the purpose of analyzing low probability of intercept radar signals. Based on the research performed for this paper (the novel direct comparison of the Wigner Ville Distribution versus the Choi Williams Distribution for the signal analysis of low probability of intercept triangular modulated FMCW radar signals) it was shown that the Wigner Ville Distribution by-and-large outperformed the Choi Williams Distribution for analyzing these low probability of intercept radar signals - for reasons brought out in the discussion section above. More accurate characterization metrics could well translate into saved equipment and lives.

Future plans include continuing to analyze low probability of intercept radar waveforms (such as the frequency hopping and the triangular modulated FMCW), using additional time-frequency analysis techniques.

\section{REFERENCES RÉFÉRENCES REFERENCIAS}

1. [ANJ09] Anjaneyulu, L., Murthy, N., Sarma, N., A Novel Method for Recognition of Modulation Code of LPI Radar Signals. International Journal of Recent Trends in Engineering, Vol.1,No.3, pp.176-180, May 2009.
2. [BOA03] Boashash, B., Time Frequency Signal Analysis and Processing: A Comprehensive Reference. Elsevier, Oxford, England, 2003.

3. [CHO89] Choi, H., Williams, W., Improved TimeFrequency Representation of Multicomponent Signals Using Exponential Kernels. IEEE Transactions on Acoustics, Speech, and Signal Processing. Vol.37,pp.862-871, June 1989.

4. [GUL07] Gulum, T., Autonomous Non-Linear Classifications of LPI Radar Signal Modulations. Thesis, Naval Postgraduate School, Monterey, CA, 2007.

5. [GUL08] Gulum, T., Pace, P., Cristi, R., Extraction of Polyphase Radar Modulation Parameters Using a Wigner-Ville Distribution-Radon Transform. IEEE International Conference on Acoustics, Speech, and Signal Processing, Las Vegas, NV, April 2008.

6. [HAN00] Han, S., Hong, H., Seo, D., Choi, J., Target Position Extraction Based on Instantaneous Frequency Estimation in a Fixed-Reticle Seeker. Opt. Eng., Vol. 39, pp. 2568-2573, September 2000.

7. [HLA92] Hlawatsch, F., Boudreaux-Bartels, G.F., Linear and Quadratic Time-Frequency Signal Representations. IEEE Signal Processing Mag., Vol. 9, No.2,pp.21-67,April1992.

8. [LIA09] Liang, Y., Zhang, L., Xing, M., Bao, Z., High-Speed Ground Moving Target Detection Research Using Triangular Modulation FMCW. Front. Electr. Electron. Eng., China, 4(2), pp. 127133, 2009.

9. [LIW08] Li, W., Dan, M., Wang, X., Li, D., Wang, G., Fast Estimation Method and Performance Analysis of Frequency Modulation Rate Via RAT. Proceedings of the 2008 IEEE International Conference on Information and Automation, Zhangjiajie, China, pp. 144-147, June 20-23, 2008.

10. [LIX08] Li, X., Bi, G., A New Reassigned TimeFrequency Representation. $16^{\text {th }}$ European Signal Processing Conference, Lausanne, Switzerland, pp. 1-4, August 25-29, 2008.

11. [LIY03] Li, Y., Xiao, X., Recursive Filtering Radon-Ambiguity Transform Algorithm for Detecting Multi-LFM Signals. Journal of Electronics (China), Vol.20,No.3,pp.161-166,May 2003.

12. [MIL02] Milne, P., Pace, P., Wigner Distribution Detection and Analysis of FMCW and P-4 Polyphase LPI Waveforms. Proceedings of ICASSP, Orlando, FL, pp. 3944-3947, 2002.

13. [OZD03] Ozdemir, A., Time-Frequency Component Analyzer. Dissertation, Bilkent University, Ankara, Turkey, Sept. 2003.

14. [PAC09] Pace, P., Detecting and Classifying Low Probability of Intercept Radar. Artech House, Norwood, MA, 2009. 
15. [PAP95] Papandreou, A., Boudreaux-Bartels, G. F., Kay, S., Detection and Estimation of Generalized Chirps Using Time-Frequency Representations. 1994 Conference Record of the Twenty-Eighth Asilomar Conference on Signals, Systems and Computers, pp. 50-54, 1994.

16. [QIA02] Qian, S., Introduction To TimeFrequency and Wavelet Transforms. Prentice Hall, Upper River, NJ, 2002.

17. [RAN01] Rangayyan, R., Krishnan, S., Feature Identification in the Time-Frequency Plane by Using the Hough-Radon Transform. Pattern Recognition, Vol. 34, pp. 1147-1158, 2001.

18. [STE96] Stephens, J., Advances in Signal Processing Technology for Electronic Warfare. IEEE AES Systems Magazine, pp. 31-38, November 1996.

19. [UPP08] Upperman, T., ELINT Signal Processing Using Choi-Williams Distribution on Reconfigurable Computers for Detection and Classification of LPI Emitters. Thesis, Naval Postgraduate School, Monterey, CA, March 2008.

20. [WAJ08] Wang, Y., Jiang, Y., Detection and Parameter Estimation of Multicomponent LFM Signal Based on the Cubic Phase Function. EURASIP Journal on Advances in Signal Processing, Vol. 2008, Article ID 743985, pp. 1-7, 2008

21. [WAN10] Wang, P., Li, H., Djurovic, I., Himed, B., Integrated Cubic Phase Function for Linear FM Signal Analysis. IEEE Transactions on Aerospace and Electronic Systems, to appear, 2010.

22. [WEI03] Wei, G., Wu, S., Mao, E., Analysis of Multicomponent LFM Signals Using TimeFrequency and the Gray-Scale Inverse Hough Transform. IEEE Workshop on Statistical Signal Processing, pp. 190-193, September 28 - October 1, 2003.

23. [WIL06] Wiley, R., ELINT: The Interception and Analysis of Radar Signals. Artech House, Norwood, MA, 2006.

24. [WIL92] Williams, W., Jeong, J., Reduced Interference Time-Frequency Distributions. TimeFrequency Signal Analysis: Methods and Applications (B. Boashash, ed.), LongmanCheshire/Wiley, Melbourne/N.Y., 1992.

25. [WON09] Wong, K., Davidson, T., Abelkader, S., Detection of Low Probability of Intercept Radar Signals. Defence R\&D Canada - Ottawa Contract Report 2009-142, September 2009.

26. [XIA99] Xia, X., Chen, V., A Quantitative SNR Analysis for the Pseudo Wigner-Ville Distribution. IEEE Transactions on Signal Processing, Vol.47, No.10,pp.2891-2894,October, 1999. 